\title{
The Capacity to within a constant gap of the Gaussian Half-Duplex Relay Channel
}

\author{
Martina Cardone ${ }^{\dagger}$, Daniela Tuninetti*, Raymond Knopp ${ }^{\dagger}$, Umer Salim ${ }^{\ddagger}$ \\ $\dagger$ Eurecom, Campus SophiaTech, 450 Route des Chappes, Biot, 06410, Email: \{cardone, knopp\}@eurecom.fr \\ * University of Illinois at Chicago, Chicago, IL 60607, USA, Email: danielat@uic.edu \\ $\ddagger$ Intel Mobile Communications, Sophia Antipolis, 06560, France, Email: umer.salim@intel.com
}

\begin{abstract}
This paper studies the Gaussian half duplex relay channel, where the relay node can not transmit and receive at the same time. The main contribution lies in showing that both Partial-Decode-Forward and Compress-Forward achieve the CutSet upper bound to within a constant gap regardless of the channel parameters. This provides a closed form characterization of the Generalized Degrees-of-Freedom (gDoF) of the channel, which for certain channel parameters is strictly smaller than the gDoF of the full duplex channel. Half duplex channels can convey information through the random switch between the receive and retransmit phases; this work shows numerically that random switch achieves larger rates compared to deterministic switch, which is usually considered in the literature.
\end{abstract}

\section{INTRODUCTION}

Cooperative transmission enhances the performance of wireless networks by increasing the data rate, enlarging the communication range and reducing transmit power compared to networks of point-to-point links. The Relay Channel (RC) represents the simplest model of cooperation in which a source transmits a message to a destination with the help of a Relay Node (RN). The RN has no own data to send and its only purpose is to assist the source in conveying data to the destination. The RN can operate in two modes: Full-Duplex (FD) and Half-Duplex (HD). The RN is said to operate in FD mode if it can receive and transmit simultaneously over the same time-frequency resource, and in HD mode otherwise. HD can be achieved for example through time or frequency division duplexing. This work assumes a Gaussian HD RC (GHD-RC) for practical reasons, such as the inability of the RN to perfectly cancel its self-interference. A comprehension of the fundamental limits of the G-HD-RC may help in designing more efficient wireless networks in the future.

Related Work. The RC was introduced by van der Meulen [1] who derived outer and inner bounds on its capacity. These bounds were significantly improved upon by Cover and ElGamal [2]: their cut-set bound and their combination of Decode-Forward (DF) and Compress-Forward (CF) remain the best known upper and lower bounds, respectively, known to date on the capacity of the general memoryless RC. In Gaussian noise and with a FD RN, the bounds of [2] are no more than 1 bit apart for any set of channel parameters [3].

The G-HD-RC was considered in [4], [5] (an exhaustive list of related publications is not given for sake of space). In [4] an outer bound based on cut-set arguments and an inner bound based on Partial-Decode-Forward (PDF) were derived (PDF generalizes DF in that the RN only decodes a part of the source message). In [5] a scheme based on CF was developed. In the above mentioned works, the listen/transmit phases of the RN are assumed to be fixed and no performance guarantee in terms of constant gap between inner and outer bounds is given.

Kramer [6] showed that the HD constraint can be easily incorporated within the general memoryless FD framework and demonstrated that higher rates can be achieved by considering a random switch at the RN compared to deterministic switch. In [7], the authors showed that a Quantize-MapForward scheme based on lattice codes is optimal to within 5 bits, by also accounting for random switch in the outer bound. In [8] we followed the approach of [6] and developed a simple scheme that achieves the $\mathrm{gDoF}$ upper bound and the capacity to within 3 bits. Our achievable scheme was inspired by the analysis of the binary-valued linear deterministic approximation of the G-HD-RC at high SNR [3] and consisted of a three-message superposition coding scheme with slotby-slot stripping decoding. In [8] we mentioned that more complex achievable schemes, such as PDF or CF, should likely reduce the 3 bits gap; the proof of this conjecture is the main contribution of this paper.

Contributions. This work focuses on the G-HD-RC and strengthens the results of [8] and [7]. The main novelty of this work consists in showing that the capacity of the G-HD$\mathrm{RC}$ can be achieved to within 1 bit with PDF and to within 1.61 bits with CF. We first incorporate the HD constraint into the general memoryless FD framework [6], thus avoiding to develop a separate theory for HD RCs; then, we prove that both PDF and CF achieve the gDoF of the channel; finally, we show that they are to within a constant gap of the cut-set upper bound, regardless of the channel parameters. The key ingredients to show the reduced gap compared to [8] are more complex achievable schemes and more careful bounding steps. We conclude by numerically showing that a random switch at the $\mathrm{RN}$ achieves higher rates compared to deterministic switch traditionally considered in the literature.

Paper Organization. Section II describes the channel model and defines the metrics that will be used. Section III upper bounds the cut-set bound and Section IV lower bounds the PDF and CF bounds. These bounds are used in Section V to analytically derive the $\mathrm{gDoF}$ and characterize the capacity to within a constant gap. Section VI concludes the paper. 


\section{System Model AND BACKGROUND}

Memoryless RC Model. A RC consists of two input alphabets $\left(\mathcal{X}_{s}, \mathcal{X}_{r}\right)$, two output alphabets $\left(\mathcal{Y}_{r}, \mathcal{Y}_{d}\right)$ and a memoryless transition probability $P_{Y_{r}, Y_{d} \mid X_{s}, X_{r}}$. The source has a message $W$ uniformly distributed on $\left[1: 2^{N R}\right]$ for the destination, where $N$ denotes the codeword length and $R \geq 0$ the transmission rate measured in bits per channel use (logarithms are in base 2). At time $i, i \in[1: N]$, the source maps the message $W$ into a channel input symbol $X_{s, i}(W)$, and the relay maps its past channel observations into a channel input symbol $X_{r, i}\left(Y_{r}^{i-1}\right)$. At time $N$, the destination makes an estimate of the message $W$ based on all its channel observations as $\widehat{W}\left(Y_{d}^{N}\right)$. The capacity is the largest rate $R$ such that $\mathbb{P}[\widehat{W} \neq W] \rightarrow 0$ as $N \rightarrow+\infty$.

G-HD-RC Model. Inspired by [6] and with a slight abuse of notation, let the channel input of the $\mathrm{RN}$ in the above memoryless RC model be the pair $\left(X_{r}, M_{r}\right)$, where $X_{r} \in \mathcal{X}_{r}$ as before and $M_{r} \in\{0,1\}$ is the state random variable that indicates whether the $\mathrm{RN}$ is in receive-mode $\left(M_{r}=0\right)$ or in transmit-mode $\left(M_{r}=1\right)$. The single-antenna G-HD-RC is described by the input/output relationship

$$
\left[\begin{array}{l}
Y_{r} \\
Y_{d}
\end{array}\right]=\left[\begin{array}{cc}
1-M_{r} & 0 \\
0 & 1
\end{array}\right]\left[\begin{array}{cc}
\sqrt{C} & \star \\
\sqrt{S} & \sqrt{I} \mathrm{e}^{\mathrm{j} \theta}
\end{array}\right]\left[\begin{array}{cc}
1 & 0 \\
0 & M_{r}
\end{array}\right]\left[\begin{array}{l}
X_{s} \\
X_{r}
\end{array}\right]+\left[\begin{array}{l}
Z_{r} \\
Z_{d}
\end{array}\right],
$$

where the inputs are subject to the average power constraints $\mathbb{E}\left[\left|X_{i}\right|^{2}\right] \leq 1, i \in\{s, r\}$, the channel parameters $(S, C, I, \theta) \in \mathbb{R}_{+}^{4}$ are constant and therefore known to all terminals (here $\star$ indicates the channel gain that does not affect the capacity region because the $\mathrm{RN}$ can remove the contribution of its transmitted signal $X_{r}$ from its received signal $Y_{r}$; then two of the remaining channel gains can be taken to be real-valued because the nodes can compensate for the phase of one channel gain), and the noises are proper-complex Gaussian random variables with parameter $Z_{i} \sim \mathcal{N}(0,1), i \in\{r, d\}$. In the following we shall assume, not without loss of generality though, that the noises are independent.

Performance metrics. The capacity of the G-HD-RC, indicated with $\mathrm{C}(S, I, C)$, is unknown. Here we make progress toward determining $\mathrm{C}(S, I, C)$ by first establishing the $\mathrm{gDoF}$ of the G-HD-RC and then characterizing $\mathrm{C}(S, I, C)$ to within a constant gap. The gDoF is defined as

$$
\mathrm{d}(\alpha, \beta):=\lim _{S \rightarrow+\infty} \frac{\mathrm{C}\left(S=S^{1}, I=S^{\alpha}, C=S^{\beta}\right)}{\log (1+S)},
$$

where $(\alpha, \beta) \in \mathbb{R}_{+}^{2}$ are constants. Note that $\mathrm{d}(\alpha, \beta) \geq 1$ where $\mathrm{d}=1$ is the $\mathrm{gDoF}$ of a point-to-point channel without a RN.

At finite $S$ the capacity is said to be known to within GAP bits if one can show rates $R^{(\mathrm{in})}$ and $R^{(\text {out })}$ such that

$$
R^{\text {(in) }} \leq \mathrm{C}(S, I, C) \leq R^{\text {(out) }} \leq R^{\text {(in) }}+\mathrm{GAP} \log (2) .
$$

\section{OUTER BOUND}

This section is devoted to the derivation of the upper bound that we shall use in Section $\mathrm{V}$ to obtain the gDoF and the capacity characterization to within a constant gap. From the cut-set bound [9, Th. 16.1] we have:
Proposition 1 (Cut-Set Upper Bound [8].) The capacity of the G-HD-RC is upper bounded as (3)-(5) at the top of the next page where

- in (3): the distribution $P_{X_{s}, X_{r}, M_{r}}^{*}$ (unknown) is the one that maximizes the cut-set upper bound;

- in (4): the maximization is over the set

$$
\begin{aligned}
& \gamma:=\mathbb{P}\left[M_{r}=0\right]: \gamma \in[0,1], \\
& \left|\alpha_{1}\right| \leq 1, \\
& \left(P_{s, 0}, P_{s, 1}, P_{r, 0}, P_{r, 1}\right) \in \mathbb{R}_{+}^{4}: \\
& \quad \gamma P_{u, 0}+(1-\gamma) P_{u, 1} \leq 1, u \in\{s, r\},
\end{aligned}
$$

i.e., $\gamma$ represents the fraction of time the $R N$ listens and $\mathcal{H}(\gamma):=-\gamma \log (\gamma)-(1-\gamma) \log (1-\gamma)$, and the mutual information $I_{1}, \ldots, I_{4}$ terms are defined as

$$
\begin{aligned}
I_{1} & :=\log \left(1+S P_{s, 0}\right), \\
I_{2} & :=\log \left(1+S P_{s, 1}+I P_{r, 1}+2\left|\alpha_{1}\right| \sqrt{S P_{s, 1} I P_{r, 1}}\right), \\
I_{3} & :=\log \left(1+(C+S) P_{s, 0}\right), \\
I_{4} & :=\log \left(1+\left(1-\left|\alpha_{1}\right|^{2}\right) S P_{s, 1}\right) ;
\end{aligned}
$$

- in (5): the terms $b_{1}$ and $b_{2}$ are defined as

$$
\begin{aligned}
& b_{1}:=\frac{\log \left(1+(\sqrt{I}+\sqrt{S})^{2}\right)}{\log (1+S)} \geq 1, \\
& b_{2}:=\frac{\log (1+C+S)}{\log (1+S)} \geq 1 .
\end{aligned}
$$

Proof: in order to obtain (4) from (3) we used the mutual information chain rule and the following considerations: (a) for a binary-valued random variable $M_{r}, I\left(M_{r} ; Y_{d}\right) \leq H\left(M_{r}\right)=$ $\mathcal{H}(\gamma)$ for some $\gamma:=\mathbb{P}\left[M_{r}=0\right] \in[0,1]$ and where $\mathcal{H}(\gamma)$ is the binary entropy function, and (b) by the 'Gaussian maximizes entropy' principle [9, Appendix 16A], the mutual information terms conditioned on $M_{r}$ are maximized by jointly Gaussian inputs with covariance matrix

$\left.\operatorname{Cov}\left[\begin{array}{c}X_{s} \\ X_{r}\end{array}\right]\right|_{M_{r}=\ell}=\left[\begin{array}{cc}P_{s, \ell} & \alpha_{\ell} \sqrt{P_{s, \ell} P_{r, \ell}} \\ \alpha_{\ell}^{*} \sqrt{P_{s, \ell} P_{r, \ell}} & P_{r, \ell}\end{array}\right]:\left|\alpha_{\ell}\right| \leq 1$,

for some $\left(P_{s, 0}, P_{s, 1}, P_{r, 0}, P_{r, 1}\right) \in \mathbb{R}_{+}^{4}$ satisfying the average power constraint in (14).

In order to get (5) from (4) we write the average power constraints in (14) as follows. Since the source transmits in both phases we define $P_{s, 0}=\frac{\beta}{\gamma}$ and $P_{s, 1}=\frac{1-\beta}{1-\gamma}$ for some $\beta \in[0,1]$. Since the RN transmission only affects the destination output when $M_{r}=1$, the RN must exploit all its available power when $M_{r}=1$; we therefore let $P_{r, 0}=0$ and $P_{r, 1}=\frac{1}{1-\gamma}$. Finally, by first upper-bounding each mutual information term over $\left(\beta, \alpha_{0}\right)$ for a fixed $\left(\gamma, \alpha_{1}\right)$ and then by equating the two expressions inside the min function in order to get the optimum $\gamma=\frac{b_{1}-1}{\left(b_{1}-1\right)+\left(b_{2}-1\right)}$, we get (5).

\section{LOWER BOUNDS}

The largest achievable rate for the $\mathrm{RC}$ is the combination of PDF and CF [2]. In this section we consider them separately.

From the PDF lower bound [9, Th. 16.3] we have: 


$$
\begin{aligned}
\mathrm{C}(S, I, C) & \max _{P_{X_{s}, X_{r}, M_{r}}} \min \left\{I\left(X_{s}, X_{r}, M_{r} ; Y_{d}\right), I\left(X_{s} ; Y_{r}, Y_{d} \mid X_{r}, M_{r}\right)\right\} \\
& =\left.\min \left\{I\left(M_{r} ; Y_{d}\right)+I\left(X_{s}, X_{r} ; Y_{d} \mid M_{r}\right), I\left(X_{s} ; Y_{r}, Y_{d} \mid X_{r}, M_{r}\right)\right\}\right|_{\left(X_{s}, X_{r}, M_{r}\right) \sim P_{X_{s}, X_{r}, M_{r}}^{*}} \\
& \leq \max \min \left\{\mathcal{H}(\gamma)+\gamma I_{1}+(1-\gamma) I_{2}, \gamma I_{3}+(1-\gamma) I_{4}\right\}=: r^{(\mathrm{CS})} \\
& \leq 2 \log (2)+\log (1+S)\left(1+\frac{\left(b_{1}-1\right)\left(b_{2}-1\right)}{\left(b_{1}-1\right)+\left(b_{2}-1\right)}\right) \\
\mathrm{C}(S, I, C) \geq & \min \left\{I\left(X_{s}, X_{r}, M_{r} ; Y_{d}\right)\right. \\
& \left.I\left(U ; Y_{r} \mid X_{r}, M_{r}\right)+I\left(X_{s} ; Y_{d} \mid X_{r}, M_{r}, U\right)\right\}\left.\right|_{\left(X_{s}, X_{r}, M_{r}\right) \sim P_{X_{s}, X_{r}, M_{r}}^{*} \text { and } U=X_{r} \text { or } U=X_{r} M_{r}+X_{s}\left(1-M_{r}\right)}, \\
\geq & \max \min \left\{I_{0}^{(\mathrm{PDF})}+\gamma I_{5}+(1-\gamma) I_{6}, \gamma I_{7}+(1-\gamma) I_{8}\right\}=: r^{(\mathrm{PDF})} \\
\geq & \log (1+S)\left(1+\frac{\left(c_{1}-1\right)\left(c_{2}-1\right)}{\left(c_{1}-1\right)+\left(c_{2}-1\right)}\right) \\
\mathrm{C}(S, I, C) \geq & { }_{P_{Q} P_{M_{r} \mid Q} P_{X_{s} \mid Q} P_{X_{r} \mid M_{r}, Q} P_{\widehat{Y}_{r} \mid X_{r}, Y_{r}, M_{r}, Q}:|Q| \leq 2} \min \left\{I\left(X_{s} ; \widehat{Y}_{r}, Y_{d} \mid Q, M_{r}, X_{r}\right),\right. \\
& \left.I_{0}^{(\mathrm{CF})}+I\left(X_{s}, X_{r} ; Y_{d} \mid M_{r}, Q\right)-I\left(Y_{r} ; \widehat{Y}_{r} \mid X_{s}, X_{r}, Y_{d}, M_{r}, Q\right)\right\} \\
\geq & \max \min \left\{\gamma I_{9}+(1-\gamma) I_{10}, \gamma I_{11}+(1-\gamma) I_{12}\right\}=: r^{(\mathrm{CF})} \\
\geq & -\log (2)+\log (1+S)\left(1+\frac{\left(c_{3}-1\right)\left(c_{4}-1\right)}{\left(c_{3}-1\right)+\left(c_{4}-1\right)}\right)
\end{aligned}
$$

Proposition 2 (PDF Lower Bound.) The capacity of the $G$ $H D-R C$ is lower bounded as in (6)-(8) at the top of this page

- in (6): we fix the input $P_{U, X_{s}, X_{r}, M_{r}}$ so that $P_{X_{s}, X_{r}, M_{r}}$ is the same distribution that maximizes the cut-set upper bound in (3) and $P_{U \mid X_{s}, X_{r}, M_{r}}$ is with either $U=X_{r}$ or $U=X_{r} M_{r}+X_{s}\left(1-M_{r}\right)$

- in (7): the maximization is over the set (12)-(14) as for the cut-set upper bound in (4), the mutual information terms $I_{5}, \ldots, I_{8}$ are

$$
\begin{aligned}
& I_{5}:=I_{1} \text { in }(15), \\
& I_{6}:=I_{2} \text { in }(16), \\
& I_{7}:=\log \left(1+\max \{C, S\} P_{s, 0}\right) \leq I_{3} \text { in (17), } \\
& I_{8}:=I_{4} \text { in }(18),
\end{aligned}
$$

and $I_{0}^{(\mathrm{PDF})}:=I\left(M_{r} ; Y_{d}\right)$ is computed from the density

$$
f_{Y_{d}}(t)=\frac{\gamma}{\pi v_{0}} \exp \left(-|t|^{2} / v_{0}\right)+\frac{1-\gamma}{\pi v_{1}} \exp \left(-|t|^{2} / v_{1}\right),
$$

with $t \in \mathbb{C}, v_{0}=\exp \left(I_{5}\right)$ and $v_{1}=\exp \left(I_{6}\right)$;

- in (8): the terms $c_{1}$ and $c_{2}$ are

$$
\begin{aligned}
& c_{1}:=\frac{\log (1+I+S)}{\log (1+S)} \geq 1, c_{1} \leq b_{1}, \\
& c_{2}:=\frac{\log (1+\max \{C, S\})}{\log (1+S)} \geq 1, c_{2} \leq b_{2} .
\end{aligned}
$$

Proof: the proof follows the same type of steps used in the proof of Proposition 1 with the difference that the bound in (8) is obtained from the one in (7) with the possibly suboptimal choice of $\beta=\gamma$.
From the CF lower bound [9, Th. 16.4] we have:

Proposition 3 (CF Lower Bound.) The capacity of the $G$ $H D-R C$ is lower bounded as in (9)-(11) at the top of this page

- in (9): $\widehat{Y}_{r}=Y_{r}+\widehat{Z}_{r}$ with $\widehat{Z}_{r} \sim \mathcal{N}\left(0, \sigma_{0}^{2}\right)$ independent of all other random variables and $I_{0}^{(\mathrm{CF})}:=I\left(M_{r} ; Y_{d} \mid Q\right)$;

- in (10): the mutual information terms $I_{9}, \ldots, I_{12}$ are

$$
\begin{aligned}
I_{9} & :=\log \left(1+S P_{s, 0}+\frac{C}{1+\sigma_{0}^{2}} P_{s, 0}\right), \\
I_{10} & :=\log \left(1+S P_{s, 1}\right), \\
I_{11} & :=\log \left(1+S P_{s, 0}\right)-\log \left(1+\frac{1}{\sigma_{0}^{2}}\right), \\
I_{12} & :=\log \left(1+S P_{s, 1}+I P_{r, 1}\right),
\end{aligned}
$$

with the optimal $\sigma_{0}^{2}:=\frac{B+1}{(1+A)^{\frac{1}{\gamma}-1}-1}$, with $A:=\frac{I P_{r, 1}}{1+S P_{s, 1}}$ and $B:=\frac{C P_{s, 0}}{1+S P_{s, 0}}$, obtained by equating the two expressions within the min in (10);

- in (11): the terms $c_{3}$ and $c_{4}$ are

$$
\begin{aligned}
c_{3} & :=c_{1} \text { in }(26), c_{3} \leq b_{1}, \\
c_{4} & :=\frac{\log \left(1+\frac{C}{1+\sigma_{0}^{2}}+S\right)}{\log (1+S)} \geq 1, c_{4} \leq b_{2} .
\end{aligned}
$$

Proof: the proof follows the same type of steps used in the proof of Proposition 1 with the difference that the bound in (10) is obtained from the one in (9) with $Q=M_{r}$, that is, $I_{0}^{(\mathrm{CF})}:=I\left(M_{r} ; Y_{d} \mid Q\right)=0$, and the bound in (11) is obtained 
from (10) with the possibly suboptimal choices of $\beta=\gamma$ and $\sigma_{0}^{2}=1$.

\section{GDOF AND CAPACITY TO WITHIN A CONSTANT GAP}

\section{A. Analytical Results}

The lower bound in (6) will be compared to the upper bound in (3) to prove that PDF with random switch achieves capacity to within $1 \mathrm{bit}$; the one in (7) with the one in (4) to prove that PDF with deterministic switch also achieves capacity to within 1 bit and for numerical evaluations; the one in (8) with the one in (5) for analytical computations, i.e., evaluation of the gDoF and gaps. Similarly, we will compare the CF lower bounds in (9)-(11) with the cut-set upper bounds in (3)-(5).

From Propositions 1, 2 and 3 we have our first main result:

Theorem 1 (gDoF.) Both PDF and CF achieve the gDoF of the $G-H D-R C$ given by [8]

$$
\mathrm{d}(\alpha, \beta)=\left\{\begin{array}{ll}
1+\frac{(\beta-1)(\alpha-1)}{(\beta-1)+(\alpha-1)} & \text { for } \alpha>1, \beta>1 \\
1 & \text { otherwise. }
\end{array} .\right.
$$

Proof: The lower bound in (8) for PDF, and the one in (11) for $\mathrm{CF}$, in the $\mathrm{gDoF}$ definition in (1) give a $\mathrm{gDoF}$ lower bound; the cut-set bound in (5) used in (1) gives a gDoF upper bound (as already done in [8]); straightforward computations show that all bounds equal the gDoF expression in (34).

Remark 1: the gDoF of the G-FD-RC is [3]

$$
\mathrm{d}^{(\mathrm{FD})}(\alpha, \beta)=\max \{1, \min \{\alpha, \beta\}\} \geq \mathrm{d}(\alpha, \beta)
$$

with equality if and only if $\min \{\alpha, \beta\} \leq 1$, that is, when direct transmission from the source to the destination is gDoFoptimal (in which case $\mathrm{d}^{(\mathrm{FD})}=\mathrm{d}=1$ ).

We next show that the upper and lower bounds used to determine the gDoF in Theorem 1 are to within a constant gap of one another for any value of $(S, C, I)$. We consider both the case of random switch (obtained by accounting for $I_{0}^{(\mathrm{PDF})}$ and $I_{0}^{(\mathrm{CF})}$ ) and of deterministic switch (obtained by setting $I_{0}^{(\mathrm{PDF})}=I_{0}^{(\mathrm{CF})}=0$ ).

Theorem 2 PDF with random switch is optimal to within 1 bit.

Proof: Consider the upper bound in (3) and the lower bound in (6). Since the term $I\left(X_{s}, X_{r}, M_{r} ; Y_{d}\right)$ is the same in both bounds, the gap is given by $\mathrm{GAP} \leq I\left(X_{s} ; Y_{r}, Y_{d} \mid X_{r}, M_{r}\right)-I\left(U ; Y_{r} \mid X_{r}, M_{r}\right)-$ $I\left(X_{s} ; Y_{d} \mid X_{r}, M_{r}, U\right)$. Next we consider two different choices for $U$, which might not necessarily be the ones that minimize the gap:

- for $C \leq S$ let $U=X_{r}$, hence

$$
\begin{aligned}
\mathrm{GAP} & \leq \mathbb{P}\left[M_{r}=0\right] I\left(X_{s} ; \sqrt{C} X_{s}+Z_{r} \mid \sqrt{S} X_{s}+Z_{d}\right) \\
& \leq \mathbb{P}\left[M_{r}=0\right] \log (1+C /(1+S)) \leq \log (2) ;
\end{aligned}
$$

- for $C>S$ let $U=X_{r} M_{r}+X_{s}\left(1-M_{r}\right)$, hence

$$
\begin{aligned}
\mathrm{GAP} & \leq \mathbb{P}\left[M_{r}=0\right] I\left(X_{s} ; \sqrt{S} X_{s}+Z_{d} \mid \sqrt{C} X_{s}+Z_{r}\right) \\
& \leq \mathbb{P}\left[M_{r}=0\right] \log (1+S /(1+C)) \leq \log (2),
\end{aligned}
$$

as claimed.

Theorem 3 PDF with deterministic switch is optimal to within 1 bit.

Proof: Consider the upper bound in (4) and the lower bound in (7) with the trivial lower bound $I_{0}^{(\mathrm{PDF})}=0$. Recall that $I_{1}=I_{5}, I_{2}=I_{6}, I_{3} \geq I_{7}$ and $I_{4}=I_{8}$, therefore

$$
\begin{aligned}
\mathrm{GAP} & \leq \max \left\{\mathcal{H}(\gamma), \gamma\left(I_{3}-I_{7}\right)\right\} \\
& \leq \max \left\{\log (2), \log \left(\frac{1+2 \max \{C, S\} P_{s, 0}}{1+\max \{C, S\} P_{s, 0}}\right)\right\} \\
& \leq \max \{\log (2), \log (2)\}=\log (2),
\end{aligned}
$$

as claimed

Remark 2: we notice that, in general, random switch does not decrease the gap. This is due to the fact that, if $\min \{C, I\} \leq S$, the information is not anymore routed through the relay. Under these channel conditions, the relay stays silent and so, using a random switch, does not lead to any improvement.

Theorem 4 CF with deterministic switch is optimal to within 1.61 bits.

Proof: With the upper bound in (4) and the lower bound in (10) we have

$$
\begin{aligned}
\mathrm{GAP} \leq \max \left\{\mathcal{H}(\gamma)+(1-\gamma) \log (2)+\gamma \log \left(1+\frac{1}{\sigma_{0}^{2}}\right)\right. \\
\left.\gamma \log \left(1+\frac{\frac{\sigma_{0}^{2}}{1+\sigma_{0}^{2}} C P_{s, 0}}{1+S P_{s, 0}+\frac{1}{1+\sigma_{0}^{2}} C P_{s, 0}}\right)\right\} \\
\leq \max \left\{\mathcal{H}(\gamma)+(1-\gamma) \log (2)+\gamma \log \left(1+\frac{1}{\sigma_{0}^{2}}\right),\right. \\
\left.\gamma \log \left(1+\sigma_{0}^{2}\right)\right\} \\
\leq \max \gamma \log \left(1+2^{\frac{\mathcal{H}(\gamma)+(1-\gamma) \log (2)}{\gamma}}\right) \leq 1.6081 \mathrm{bits},
\end{aligned}
$$

where we set $\sigma_{0}^{2}=2^{\frac{\mathcal{H}(\gamma)+(1-\gamma) \log (2)}{\gamma}}$ (so as to equate the two terms in the $\max$ ) and found that the resulting function of $\gamma \in[0,1]$ has a maximum at $\gamma=0.3855$ equal to 1.6081 .

Theorem 5 CF with random switch is optimal to within 1.61 bits.

Proof: The claim follows from Remark 2.

\section{B. Numerical Evaluations}

The gaps found in the previous subsection were obtained by manipulations of the bounds in Propositions 1, 2 and 3 so as to obtain expressions that can be easily handled analytically. We remark however that some bounding steps might be quite crude resulting in a too large gap value. To prove our point, the numerical optimization of the upper-bound $r^{(\mathrm{CS})}$ in (4) and of the lower-bound $r^{(\mathrm{CF})}$ in (10), results in a GAP $\leq 1.52$ bits, which is smaller than the analytical gap in Theorem 4 of 


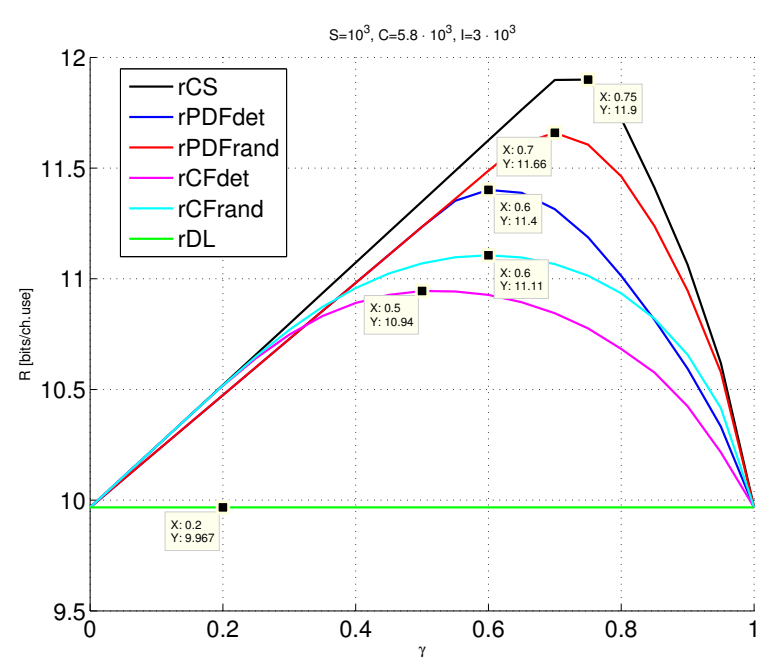

(a) $S=30 \mathrm{~dB}, \mathrm{C}=37.63 \mathrm{~dB}, \mathrm{I}=34.77 \mathrm{~dB}$.

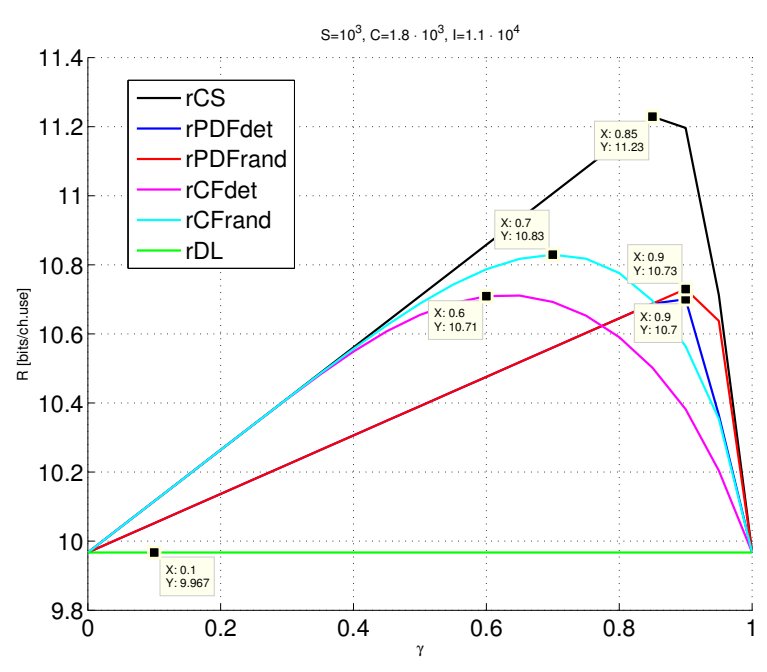

(b) $S=30 \mathrm{~dB}, \mathrm{C}=32.55 \mathrm{~dB}, \mathrm{I}=40.41 \mathrm{~dB}$.

Fig. 1. Comparison between outer and lower bounds for the G-HD-RC.

1.61 bits. Notice that we used Gaussian inputs conditioned on the RN's state $M_{r}$; this might not be an optimal choice when the RN employs random switch; other input distributions might lead to a smaller gap.

Fig. 1(a) shows the rates achieved by using the different achievable schemes presented in the previous section for $S=$ $30 \mathrm{~dB}, C=37.63 \mathrm{~dB}, I=34.77 \mathrm{~dB}$ :

- upper bound: black curve, obtained by optimizing $r^{(\mathrm{CS})}$ in (4), gives $C \leq 11.90$ bits;

- PDF with random switch: red curve, obtained by optimizing $r^{(\mathrm{PDF})}$ in (7) with the actual value of $I_{0}^{(\mathrm{PDF})}$, gives $\mathrm{C} \geq 11.66$ bits;

- PDF with deterministic switch: blue curve, obtained by optimizing $r^{(\mathrm{PDF})}$ in (7) with $I_{0}^{(\mathrm{PDF})}=0$, gives $\mathrm{C} \geq$ 11.40 bits;

- CF with random switch: cyan curve, obtained by optimizing $r^{(\mathrm{CF})}$ in (10) with the actual value of $I_{0}^{(\mathrm{CF})}$, gives $C \geq 11.11$ bits;

- CF with deterministic switch: magenta curve, obtained by optimizing $r^{(\mathrm{CF})}$ in (10), which has $I_{0}^{(\mathrm{CF})}=0$ gives $C \geq 10.94$ bits;

- direct link transmission: green curve, obtained without using the $\mathrm{RN}$, gives $\mathrm{C} \geq 9.97$ bits.

We observe that: (i) PDF with random switch achieves the largest rate among all achievable schemes, and (ii) PDF with deterministic switch outperforms the $\mathrm{CF}$ with random switch; this shows that exploiting the randomness into the switch at the RN increases the rate performance [6]. Similar observations hold for Fig. 1(b), where the channel conditions are such that $\mathrm{CF}$ outperforms PDF (cyan curve versus red curve and magenta curve versus blue curve).

\section{CONCLUSIONS}

In this work we studied the G-HD-RC and we made progress towards deriving its capacity by showing that PDF with random switch is optimal to within 1 bit regardless of the channel parameters. Moreover we showed, through numerical evaluations, that the system achieves higher rates with random switch at the RN compared to deterministic switch.

\section{ACKNOWLEDGMENT}

The work of D. Tuninetti was partially funded by NSF under award number 0643954 and thanks to the generous support of Telecom-ParisTech, Paris France, while the author was on a sabbatical leave at the same institution. Eurecom's research is partially supported by its industrial partners: BMW Group Research \& Technology, IABG, Monaco Telecom, Orange, SAP, SFR, ST Microelectronics, Swisscom and Symantec. The research at Eurecom leading to these results has received funding from the EU FP7 grant agreement CONECT $n^{\circ}$ 257616. The research at IMC leading to these results has received funding from the EU FP7 grant agreement iJOIN $n^{\circ}$ 317941.

\section{REFERENCES}

[1] E. C. van der Meulen, "Three-terminal communication channel," $A d v$. Appl. Probab., vol. 3, pp. 120-154, 1971.

[2] T. Cover and A.E. Gamal, "Capacity theorems for the relay channel," IEEE Trans. on Info. Theory, vol. 25, no. 5, pp. 572 - 584, Sep. 1979.

[3] A.S. Avestimehr, Wireless network information flow: a deterministic approach, Ph.D. thesis, EECS Department, University of California, Berkeley, Oct. 2008.

[4] A. Host-Madsen, "On the capacity of wireless relaying," in Proceedings IEEE VTC 2002, vol. 3, pp. 1333 - 1337.

[5] A. Host-Madsen and J. Zhang, "Capacity bounds and power allocation for wireless relay channels," IEEE Trans. on Info. Theory, vol. 51, no. 6 , pp. $2020-2040$, June 2005.

[6] G. Kramer, "Models and theory for relay channels with receive constraints," in Allerton 2004, pp. 1312-1321.

[7] A. Özgür and S.N. Diggavi, "Approximately achieving gaussian relay network capacity with lattice codes," arxiv:1005.1284, 2010.

[8] M. Cardone, D. Tuninetti, R. Knopp, and U. Salim, "Gaussian half-duplex relay channels: Generalized degrees of freedom and constant gap result," to appear in ICC 2013, extended version available on arXiv/1301.5522.

[9] A. El Gamal and Y.H. Kim, Network Information Theory, Cambridge Univ. Press,, Cambridge U.K., 2011. 